\title{
Investigation of the Static Breakdown Voltage of the Lubricating Film in a Mechanical Ball Bearing
}

\author{
Abhishek Joshi, Jörgen Blennow \\ High Voltage Engineering \\ Department of Materials and Manufacturing Technology \\ Chalmers University of Technology \\ SE - 41296 Gothenburg, Sweden
}

\begin{abstract}
In this paper, the current pulse (discharge) activity in a small bearing is investigated when current-limited DC voltages of different levels are applied to the shaft under varying rotational speeds and mechanical loads. The measurements show that the inception voltage of discharges in the bearing is higher than the extinction voltage. However, when the discharge activity has been intense the bearing exhibits difficulties to recover its insulating properties when the voltage is decreased to a low voltage level and thus the discharge activity continues. The measurements also show that at low voltages the bearing acts as an insulator but if the voltage is increased the bearing starts to act as a conductor. This transition occurs during a narrow voltage interval. The time interval between two consecutive current pulses decreases significantly with increasing shaft voltage.
\end{abstract}

\section{Introduction}

The mechanical bearing constitutes a key component for the reliability of a wind turbine. Understanding of how bearing damages arise and how they can be prevented is therefore of great importance. One important type of bearing damage is of electrical origin and is caused by a current passing through the lubricating film. If the voltage across the bearing exceeds a certain threshold voltage, the lubricating film will lose its insulating properties. This result in a current, sometime referred to as Electrical Discharge Machining (EDM) current [1], which will cause mechanical damages (e.g. crater formation) on both the surface of the ball and on the raceways along with a chemical decomposition of the lubricant. The phenomenon as such is not new, but after the introduction of Insulated Gate Bipolar Transistor (IGBT) switching according to the Pulse Width Modulation (PWM) scheme, the problem has become more severe.

The EDM currents have been discovered to be the main cause of premature failure within bearings of PWM inverter-fed drives. A systematic investigation of different parameters of the drive system and their influence on bearing currents revealed that the size of the motor plays a major role in the bearing current phenomenon [1]. The presence of a bearing voltage of given amplitude is an essential but not sufficient condition for the appearance of EDM current [2].

In this paper we investigate the current pulse (discharge) activity in a small bearing when current-limited DC voltages of different levels are applied to the shaft under varying rotational speeds and mechanical loads. The reason for using a current-limited voltage source is that the aim of the present study is to investigate the voltage at which the insulating properties of the bearing breaks down and not the effects caused by the current that follows upon a breakdown.

\section{Method}

\subsection{Test setup}

The studies of the breakdown voltage of a bearing exposed to a DC voltage were performed in a test-rig, fig. 1, [3]. The bearing under test, an SKF bearing 608 having 7 balls and an outer diameter of $22 \mathrm{~mm}$, was mounted on a shaft together with a hybrid bearing, SKF hybrid bearing 608 , with ceramic balls.

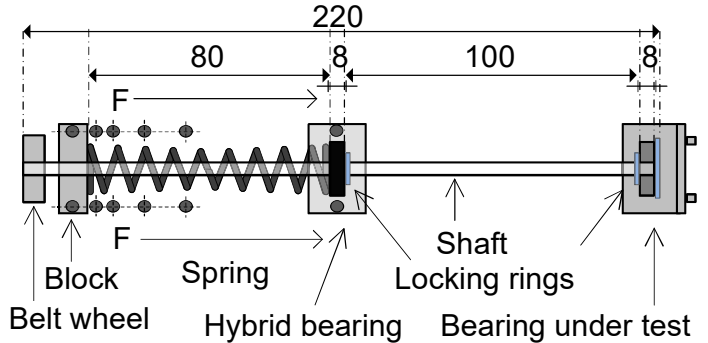

Fig. 1 - Setup of the test-rig. Measures in $\mathrm{mm}$.

The insulating ceramic roller balls in the hybrid bearing gives control over in which bearing discharges take place. The shaft is brought into rotation through a belt wheel and an insulating belt driven by a permanent magnet motor. A grounded U-girder, electrically insulated from the bearing housing, provides a solid foundation to the setup. The test voltage is applied to the shaft by means of a slip ring contact. Various levels of mechanical load can be applied by changing the position of the block. The axial load can be varied between $4 \mathrm{~N}$ and $40 \mathrm{~N}$ [3]. The radial load is approximately $1 \mathrm{~N}$ on each bearing. The tests are performed at room temperature. 


\subsection{Measurement methods}

The DC test voltage, in the range of 0 to $15 \mathrm{~V}$, is applied to the shaft through a current limiting resistance $\left(\mathrm{R}_{\text {limiting }}=981 \pm 2.5 \Omega\right)$, fig 2 . The reason for this is that the aim of the present study is to investigate the breakdown voltage and not the effects caused by the current that follows upon a breakdown. The current through the bearing is recorded using a data acquisition card (NI USB 6251) that measures the voltage across a resistive shunt $\left(R_{\text {shunt }}=81 \pm 0.5 \Omega\right)$ connected between the bearing under test and ground. The resistance value is chosen so that the pulses recorded clearly can be distinguished from the noise level $(5 \mathrm{mV})$. The data acquisition card offers a maximum aggregated sampling rate of $1 \mathrm{MS} / \mathrm{s}$. Since we use two channels, the sampling rate will be $500 \mathrm{kS} / \mathrm{s}$ per channel which corresponds to one data point sampled at every $2 \mu \mathrm{s}$. As a complement, an oscilloscope (Tektronix DPO 4034) with significantly higher sampling rate, $2.5 \mathrm{GS} / \mathrm{s}$, is used for detailed studies, fig. 3 .

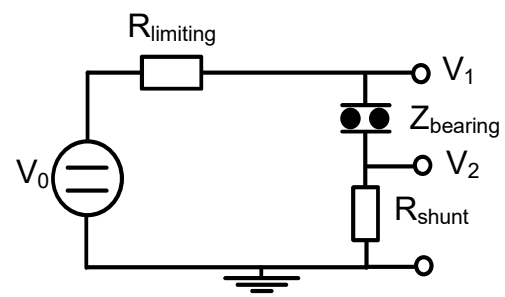

Fig. 2 - Electrical circuit model of the test setup

The current flowing through the bearing is obtained as $\mathrm{I}_{\text {bearing }}=\mathrm{V}_{2} / \mathrm{R}_{\text {shunt }}$. The voltage across the bearing can be calculated as $V_{1}-V_{2}$. Two types of DC voltage source are used: a stabilized DC power supply (CE-BIT LK 31) when the voltage needed to be easily adjusted or a set of dry cells $(\mathrm{NiMH}$ rechargeable AA, $1700 \mathrm{mAh}$ ) when a "pure" DC voltage is aimed at.

\subsection{Analysis of bearing current}

Analysis of the bearing current is performed at varying operating conditions such as different shaft voltages, rotational speeds and mechanical loads. When the bearing loses its insulating properties a current will flow through it due to the applied DC shaft voltage, fig. 3. The amplitude of the current is limited by the current limiting resistor $\mathrm{R}_{\text {limiting. After some microseconds the }}$ bearing ceases to conduct and the voltage across the bearing recovers.

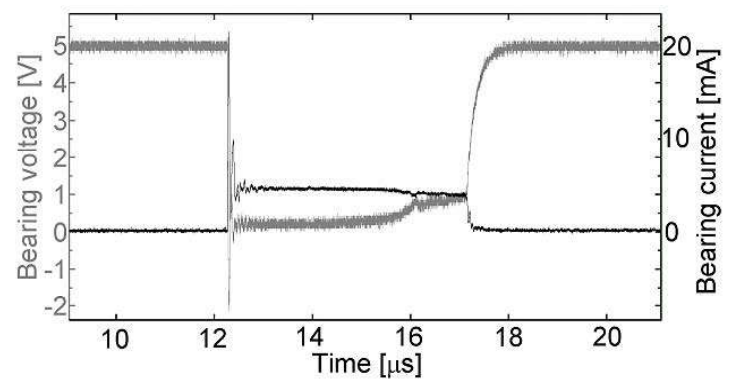

Fig. 3 - Oscilloscope trace showing the bearing current (black) and the corresponding drop in voltage across bearing (grey).
Depending on rotational speeds and mechanical loads, a minimum bearing voltage is required to initiate current pulse activity. This voltage we denote inception voltage. If current pulse activity has been initiated and the applied voltage is decreased the discharge activity will cease at a certain voltage. This voltage we denote extinction voltage.

The varying time interval between two consecutive current pulses is denoted $\Delta \mathrm{t}(\mu \mathrm{s})$, fig. 4 , and this property is below used to describe the discharge activity at different conditions. The data from $\Delta \mathrm{t}$-measurements is presented as number of intervals per second. Attempts to analyze the awaiting times to puncture of lubrication film have been presented in [2]. Also the pulse duration is a characteristic quantity which is used for describing the fraction of time (in \%) that the bearing conducts current during different conditions. A threshold of $1 \mathrm{~mA}$ is chosen to exclude effects from noise.

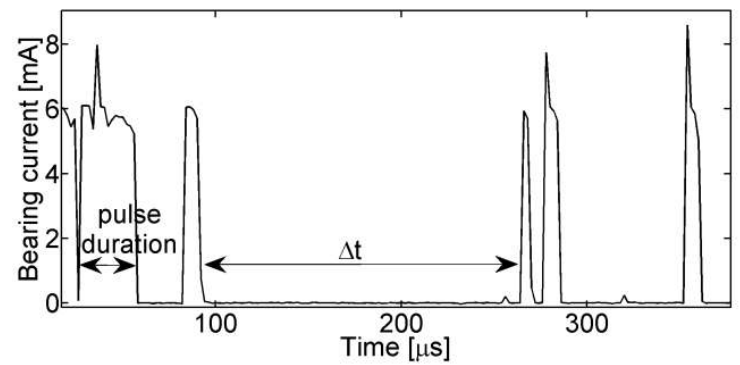

Fig. 4 - Definition of time interval, $\Delta t$, between bearing current pulses and pulse duration.

\section{Results and discussion}

The current pulse activity in the bearing as well as the inception and extinctions voltages were studied during a step-wise changed DC shaft voltage sequence under which both voltage and current were recorded. In these tests, the stabilized DC power supply was used. Since the tests in some cases were rather long (up to one hour) current pulses are presented as a sequence of 30 second windows. The measurements were conducted at a rotational speed of $3000 \mathrm{rpm}$ and a mechanical load of $40 \mathrm{~N}$. Before starting each test the shaft was grounded and left to rest at standstill for a period of six hours in between tests. In the first test, the shaft voltage was step-wise increased to $6 \mathrm{~V}$ and then step-wise decreased to $1 \mathrm{~V}$ where the voltage was kept, fig. 5. Each step lasted for approximately 10 seconds.
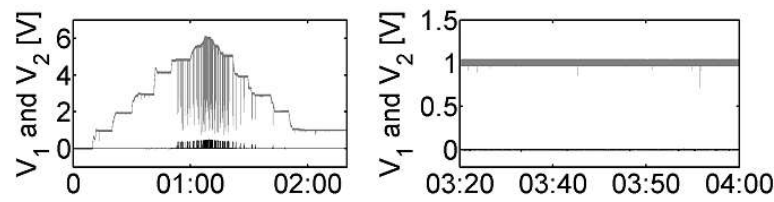

Fig. 5 - Bearing current pulse activity (black) for a voltage sequence (grey) with maximum $6 \mathrm{~V}$ DC. Inception voltage $5 \mathrm{~V}$ and extinction voltage $2 \mathrm{~V}$. Time in minutes:seconds format.

The inception voltage for current pulse activity through the bearing is approximately $5 \mathrm{~V}$ while the extinction 
voltage is significantly lower, approximately $2 \mathrm{~V}$. Thus the insulating properties of the bearing recover after that the voltage step-wise is decreased. In the second test, fig. 6 , the shaft voltage was step-wise increased to $8 \mathrm{~V}$ and then step-wise decreased to $1 \mathrm{~V}$ where the voltage was kept for 15 minutes.

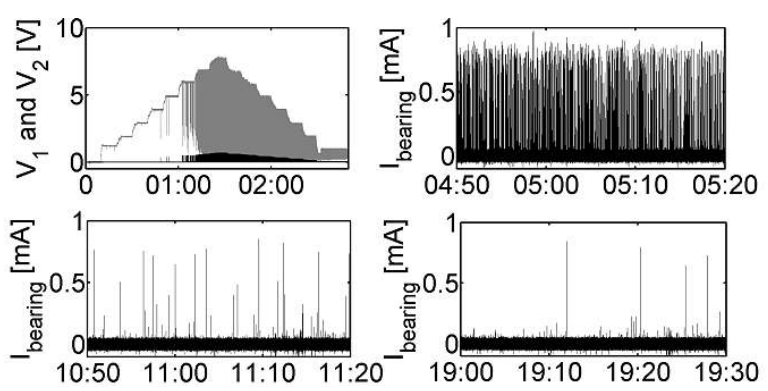

Fig. 6 - Bearing current pulse activity (black) for a voltage sequence (grey) with maximum $8 \mathrm{~V}$ DC. Inception voltage $4 \mathrm{~V}$ and extinction voltage below $1 \mathrm{~V}$. Time in minutes:seconds format.

If the diagrams in fig. 5 and fig. 6 are compared it is obvious that in fig. 6 , there are more current pulses and they also remain at lower voltage levels when the voltage is decreased. After 15 minutes with $1 \mathrm{~V}$ applied there are still bearing current pulses and thus the insulating properties of the bearing are not fully recovered. In the third test, fig. 7, the voltage was stepwise increased to $8 \mathrm{~V}$ and then step-wise decreased to $2 \mathrm{~V}$ where it was kept for one hour.
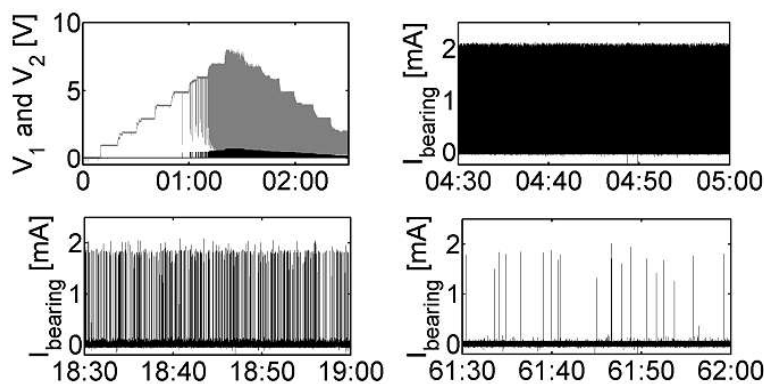

Fig. 7 - Bearing current pulse activity (black) for a voltage sequence (grey) with maximum $8 \mathrm{~V} \mathrm{DC}$. When the voltage is kept as $2 \mathrm{~V}$ the bearing current pulses continue for more than hour. Time in minutes:seconds format.

Figure 7 shows that the bearing current pulses continue for more than one hour. Thus the bearing exhibits difficulties to recover its insulating properties if a $2 \mathrm{~V}$ shaft voltage is maintained. Upon repeating tests immediately after the tests above $(<5 \mathrm{~min})$, the inception voltage was found to be $2 \mathrm{~V}$ instead of $5 \mathrm{~V}$. This might indicate that the lubricant has undergone some kind of electrochemical reaction affecting its conductivity which is supported by [4]. If the bearing is allowed to rest without voltage it recovers its insulating properties after some time.

The bearing current pulse activity was also studied through measurements of time interval between two consecutive current pulses, $\Delta \mathrm{t}(\mu \mathrm{s})$, and measurements of pulse duration. Current pulse traces were recorded and the time intervals between two consecutive pulses were evaluated. The occurrence of theses intervals is presented in histograms as number of insulation breakdowns per second representing a certain time interval (y-axis) for different time intervals $\Delta \mathrm{t}$ (x-axis). The tests were performed at three different rotational speeds (1000, 2000 and $3000 \mathrm{rpm}$ ), two different mechanical loads (4 and $40 \mathrm{~N}$ ) and four different voltage levels $(4.0,6.6,7.9$ and $10.6 \mathrm{~V})$. In these tests dry cells were used as DC source. The results from low load conditions $(4 \mathrm{~N})$ are presented in fig. 8 and 9. Fig. 8 shows that at a rotational speed of $3000 \mathrm{rpm}$, the time intervals between two consecutive pulses decrease significantly with increasing shaft voltage. The number of insulation breakdowns per second increases with shaft voltage.

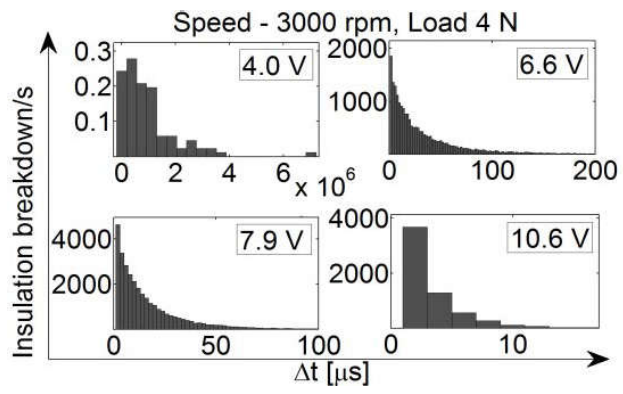

Fig. 8 - Histogram of number of insulation breakdown per second on $\mathrm{y}$-axes for different $\Delta \mathrm{t}$ on $\mathrm{x}$-axes at high speed of $3000 \pm 20 \mathrm{rpm}$ and low load of $4 \mathrm{~N}$.

When the rotational speed of the bearing is reduced to $2000 \mathrm{rpm}$, fig. 9, the times between consecutive discharges have decreased significantly compared to at $3000 \mathrm{rpm}$ indicating more frequent passage of current due to frequent asperity contacts [4].

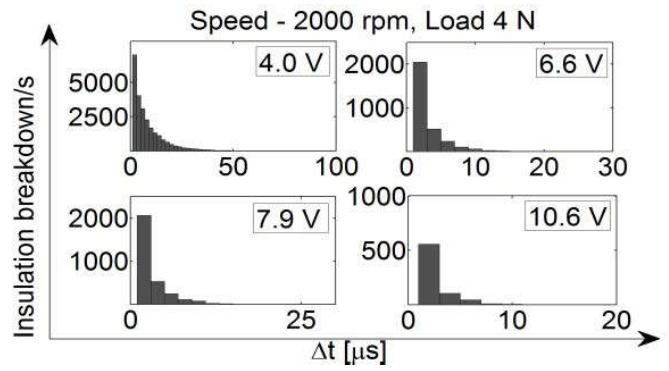

Fig. 9 - Histogram of number of insulation breakdown per second on $\mathrm{y}$-axes for different $\Delta \mathrm{t}$ on $\mathrm{x}$-axes at medium speed of $2000 \pm 20 \mathrm{rpm}$ and low load of $4 \mathrm{~N}$.

At a mechanical load of $40 \mathrm{~N}$ and a rotational speed of $3000 \mathrm{rpm}$ (not presented here due to limitation in space), the bearing discharge repetition rate is in order of few milliseconds at $4.0 \mathrm{~V}$ level. This is further reduced to a few microseconds at higher voltages. At $40 \mathrm{~N}$ and a rotational speed of $2000 \mathrm{rpm}$, the discharge repetition rate is in the order of a few $10 \mathrm{~s}$ of microseconds, but the number of insulation breakdowns per second reduces as voltage increases. This reduction in number of insulation breakdown per second is due to 
the fact that the bearing conducts current more than $90 \%$ of the time, i.e. longer pulse durations.

At a rotational speed of $1000 \mathrm{rpm}$, a constant current channel is formed at all higher voltage levels and different mechanical loads, meaning fewer and longer current pulses are recorded.

Finally, a set of tests was performed to study this transition phenomenon where the bearing starts to conduct current continuously for different mechanical loads and rotational speeds. The fraction of time (in \%) at which the bearing was conducting and the number of current pulses per second were investigated. The procedure followed that the bearing was first resting at standstill and grounded for $15 \mathrm{~min}$ and then it was rotating for $15 \mathrm{~min}$ still grounded. After this, voltage was applied in steps of $1 \mathrm{~V}$ while data were recorded. Fig. 10 shows the transition from the DC voltage level where the bearing acts as an insulator to the DC voltage level where it acts as a conductor. The transition between the two stages occurs over a small increase in voltage (a few volts).

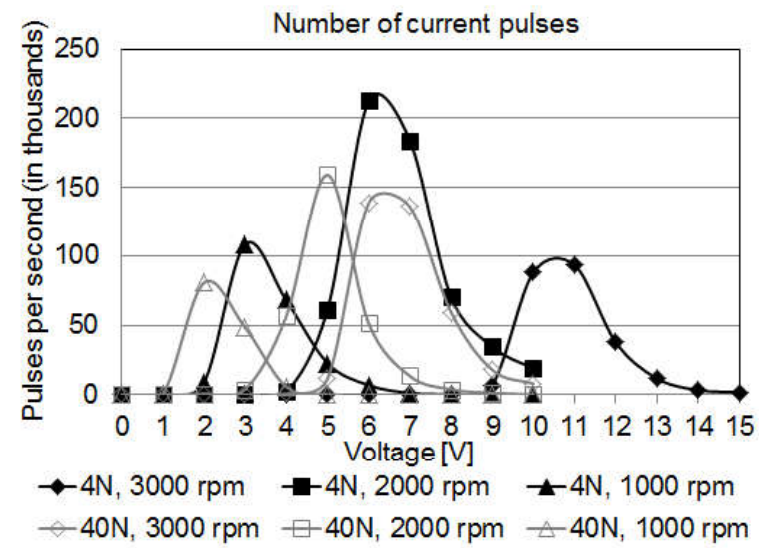

Fig. 10 - Transition of a bearing acting as an insulator at lower DC voltage levels to acting as a conductor at slightly higher voltage levels under different operating conditions.

The number of current pulses per second passing through the bearing at different mechanical loads and rotational speeds is shown in fig. 11. The curves are bell-shaped and the peaks coincide approximately with the voltage where the bearing conducts $50 \%$ of the time.

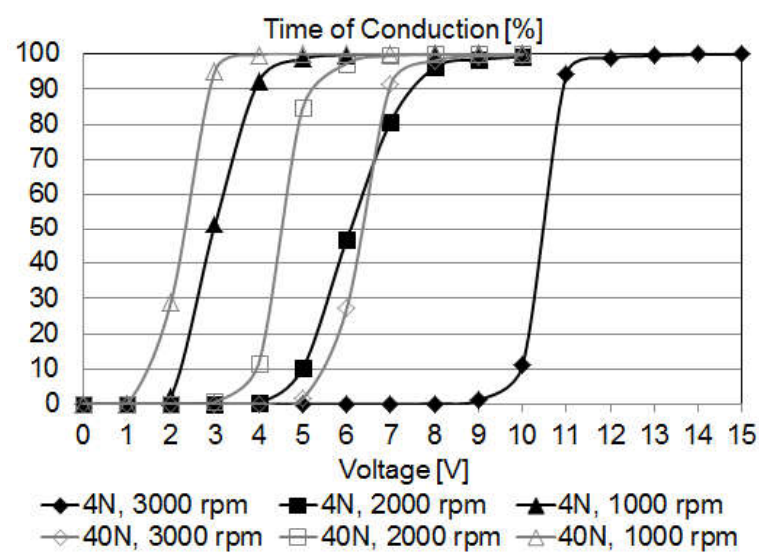

Fig. 11 - Duration of current pulses as a function of different levels of shaft voltage.

\section{Conclusions}

The measurements show that the DC inception voltage (ca $5 \mathrm{~V}$ ) of discharges in the bearing (SKF 608) is higher than the extinction voltage (ca 2 V). However, when the current pulse activity has been intense the bearing exhibits difficulties to recover its insulating properties when the DC voltage is decreased to a lower voltage level (1 to $2 \mathrm{~V}$ ) and thus the discharge activity continues. Further, the measurements also showed that at low voltages the bearing acts as an insulator but if the voltage is increased the bearing starts to act as a conductor. This transition occurs during a narrow voltage interval (a few volts). The transition voltage increases with increasing rotational speed but decreases with mechanical load.

The time interval between two consecutive current pulses decreases significantly with increasing shaft voltage.

The number of current pulses passing through the bearing per second and their duration depend on applied voltage, rotational speed and mechanical load.

\section{Acknowledgements}

This project is financed through the Swedish Wind Power Technology Centre (SWPTC). SWPTC is a research centre for design of wind turbines. The purpose of the centre is to support Swedish industry with knowledge of design techniques as well as maintenance in the field of wind power. The Centre is funded by the Swedish Energy Agency, Chalmers University of Technology as well as academic and industrial partners.

\section{References}

[1] A. Muetze, Bearing currents in inverter-fed $A C$ motors, Ph.D. thesis, Shaker Verlag, TU Darmstadt, Germany, 2004.

[2] A. Kempski, R. Smolenski, J. Bojarski "Statistical approach to discharge bearing currents", COMPEL: The International Journal for Computational and Mathematics in Electrical and Electronics Engineering, Vol. 29, Issue: 3, 2010, pp. $647-666$.

[3] M, Fyhrman and M. Edvall, Current Damages in Bearings, Master's Thesis, ISSN - 1652-8913, Chalmers University of Technology, Gothenburg, Sweden, 2007.

[4] H. Prashad, "Effect of operating parameters on the threshold voltages and impedance response of non-insulated rolling element bearings operated under action of electrical current", Wear, Vol. 117, 1987, pp. 223-240. 